\title{
Mode Selection in MU-MIMO Downlink Networks: A Physical Layer Security Perspective
}

\author{
Xiaoming Chen, Senior Member, IEEE, and Yu Zhang, Member, IEEE
}

\begin{abstract}
In this paper, we consider a homogenous multiantenna downlink network where a passive eavesdropper intends to intercept the communication between a base station (BS) and multiple secure users (SU) over Rayleigh fading channels. In order to guarantee the security of information transfer, physical layer security is employed accordingly. For such a multiple user (MU) secure network, the number of accessing SUs, namely transmission mode, has a great impact on the secrecy performance. Specifically, on the one hand, a large number of accessing SUs will arise high inter-user interference at SUs, resulting in a reduction of the capacity of the legitimate channel. On the other hand, high inter-user interference will interfere with the eavesdropper and thus degrades the performance of the eavesdropper channel. Generally speaking, the harmful interuser interference may be transformed as a useful tool of antieavesdropping. The focus of this paper is on selecting the optimal transmission mode according to channel conditions and system parameters, so as to maximize the sum secrecy outage capacity. Moreover, through asymptotic analysis, we present several simple mode selection schemes in some extreme cases. Finally, simulation results validate the effectiveness of the proposed mode selection schemes in MU secure communications.
\end{abstract}

Index Terms-Physical layer security, secrecy outage capacity, mode selection, asymptotic analysis.

\section{INTRODUCTION}

Information security is always a critical issue of wireless communications due to the open nature of wireless channels. Traditionally, information security is realized by using complex cryptography technology. In fact, information theory has proven that secure communication can be guaranteed by only exploiting the characteristics of wireless channels, e.g., fading, noise and interference, namely physical layer security [1] [2]. The essential of physical layer security is to maximize the secrecy rate, which is defined as the rate difference between the legitimate channel and the eavesdropper channel [3] [4]. If there are multiple antennas at the information source, by exploiting spatial degrees of freedom, the legitimate channel rate is increased and the eavesdropper channel rate is decreased simultaneously, so the secrecy rate can be improved significantly. Thus, physical layer security coupling with multiantenna techniques has received considerably research interests [5]-[8].

Xiaoming Chen (chenxiaomingenuaa.edu.cn) is with the College of Electronic and Information Engineering, Nanjing University of Aeronautics and Astronautics, Nanjing 210016, China. Yu Zhang (zhangyu_wing@hotmail.com) is with the Department of Information Science and Electronic Engineering, Zhejiang University, Hangzhou 310027, China.

\section{A. Related Works}

Intuitively, in multi-antenna secure networks, by transmitting the information in the null space of the eavesdropper channel, the eavesdropper can not intercept any useful information. However, in the sensing of maximizing the secrecy rate, this approach seems not to be optimal. The key is to select a transmit direction, namely beamforming, so as to achieve an optimal tradeoff between maximizing the legitimate channel capacity and minimizing the eavesdropper channel capacity [9]-[11]. In [12], the problem of optimal transmit beamforming in a MISO system was addressed by maximizing the secrecy rate. A potential drawback of the above approach lies in that the source must have full channel state information (CSI) to design the transmit beam. To alleviate the assumption, a joint power allocation and beamforming scheme was proposed based on full CSI of the legitimate channel and partial CSI of the eavesdropper channel [13]. Yet, it is difficult to obtain the CSI for the source, especially the CSI of the eavesdropper channel, because the passive eavesdropper will hide itself as good as possible. It is proved that if there is no CSI of the eavesdropper channel, the beamforming alone the direction of the legitimate channel is optimal [18]. Since the secrecy rate is jointly determined by the legitimate and the eavesdropper channel capacities, if the source has no CSI of the eavesdropper channel, it is impossible to maintain a steady secrecy rate over all realizations of fading channels. In this context, the secrecy outage capacity is adopted as a useful and intuitive metric to evaluate security, which is defined as the achievable maximum secrecy rate under the condition that the outage probability that the real transmission rate surpasses the secrecy rate is equal to a given value [14]. The secrecy outage capacity based on antenna selection is analyzed in an uncorrelated MIMO system [15] and in a correlated MIMO system [16]. Note that the assumption of full CSI for the legitimate channel at the source also seems impractical in multi-antenna systems, especially in frequency division duplex (FDD) systems. To solve this problem, limited feedback techniques are introduced into multi-antenna secure systems to convey the quantized CSI from the legitimate receiver to the source [17] [18].

Another advantage of the MIMO system lies in that it can support multiple users transmission, namely space division multiple access, so the performance is improved significantly [19]-[22]. In [23], the secrecy rate over MU-MIMO broadcasting channels was well studied. In [24], a robust beamforming scheme for MU-MIMO downlink networks was proposed by using a Bayesian approach. In MU-MIMO systems, inter- 
user interference is a pivotal factor affecting the overall performance. For a given precoding scheme, a large number of accessing users is beneficial to exploit the spatial multiplexing gain, but also arises high inter-user interference at users. Thus, it is better to select the number of accessing users, namely transmission mode, according to channel conditions and system parameters [25] [26]. Interestingly, in MU secure communications, the harmful inter-user interference can be used to interfere with the interception of the eavesdropper. Generally speaking, inter-user interference has two completely opposite functions. A detailed investigation of the impact of multiuser interference on the secrecy performance was carried out in [27], and thus multiuser scheduling was called for secrecy performance enhancement. A single user selection scheduling was proposed in [28], so the inter-user interference can be avoided completely. On the contrary, the users that the multi-antenna system can support at best was scheduled in [29], so as to improve the sum rate of the legitimate channel. In fact, the number of scheduled users should be carefully selected according to channel conditions. Therefore, it makes sense to perform mode selection in MU-MIMO downlink networks from a perspective of optimizing the performance.

\section{B. Main Contributions}

In this paper, we consider a MU-MIMO downlink network in presence of a passive eavesdropper. Considering the large CSI feedback amount for multiple legitimate channels, opportunistic space division multiple access (OSDMA) [30] [31] is adopted to exploit the MU gain due to its low complexity, small overhead and good performance. The focus of this paper is on mode selection to optimize the utility of inter-user interference in MU secure communications based on physical layer security. The major contributions of this paper can be summarized as follows:

1) We present a framework of physical layer security in MU-MIMO downlink networks with limited CSI feedback based on OSDMA, and propose to transform the harmful inter-user interference to enhance wireless security through user scheduling.

2) It is found that under different channel conditions, interuser interference plays different roles. We derive an explicit expression for secrecy outage capacity in terms of transmission mode, transmit power, user number, and channel condition. By maximizing the sum secrecy outage capacity, we obtain an adaptive mode selection scheme.

3) Through asymptotic analysis, we provide some guidelines for simple mode selection as follows:

a) At low SNR regime, maximum available mode should be adopted. Relaxing the requirement on the outage probability and increasing the number of SUs are hardly helpful to improve the secrecy performance.

b) At high SNR regime, single SU mode is the best choice and multiple-SU mode will result in zero rate. c) If the number of SUs is sufficiently large, maximum available mode can asymptotically achieve the optimal secrecy performance.

\section{Paper Organization}

The rest of this paper is organized as follows: Section II gives a brief introduction of the considered MU-MIMO downlink network employing physical layer security. Section III focuses on the analysis and the design of the mode selection scheme based on the criterion of maximizing the sum secrecy outage capacity. Through asymptotic performance analysis, we present several simple mode selection schemes in some extreme cases in Section IV. Section V presents several simulation results to validate the effectiveness of the proposed schemes, and finally Section VI concludes the whole paper.

Notations: We use bold upper (lower) letters to denote matrices (column vectors), $(\cdot)^{H}$ to denote conjugate transpose, $E[\cdot]$ to denote expectation, $\|\cdot\|$ to denote the $L_{2}$ norm of a vector, $|\cdot|$ to denote the absolute value, $(a)^{+}$to denote $\max (a, 0),\lceil a\rceil$ to denote the smallest integer not less than $a,\lfloor a\rfloor$ to denote the largest integer not greater than $a$, and $\stackrel{d}{=}$ to denote the equality in distribution. The acronym i.i.d. means "independent and identically distributed", pdf means "probability density function" and cdf means "cumulative distribution function".

\section{SYSTEM MODEL}

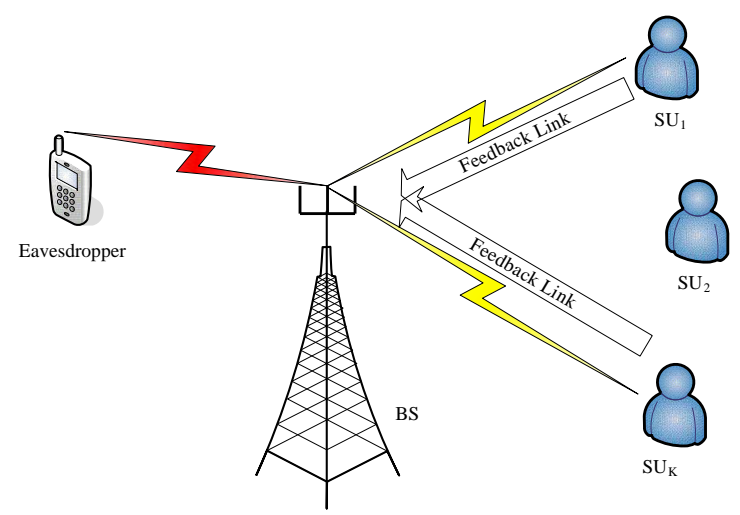

Fig. 1. A model of the MU-MIMO downlink network with physical layer security.

We consider a MU-MIMO downlink network, where a base station (BS) with $N_{t}$ antennas communicates with $K$ single antenna secure users (SU), while a single antenna eavesdropper also receives the message sent from the BS and tries to detect it. We use $\mathbf{h}_{k}$ to denote the $k$ th legitimate channel from the BS to the $k$ th SU, whose elements are i.i.d. zero mean and unit variance complex Gaussian random variables (homogeneous network in terms of users's average channels as in [29]). Similarly, we use $\alpha \mathbf{g}$ to denote the eavesdropper channel from the BS to the eavesdropper, where $\alpha$ is the relative path loss and $\mathbf{g}$ denotes the small-scale fading vector with i.i.d. zero mean and unit variance complex Gaussian distributed entries. We assume that the BS has partial instantaneous CSI 
about the legitimated channel through limited feedback and only statistical CSI about the eavesdropper channel, since the eavesdropper is passive.

The network is operated in the form of time slots. It is assumed that the channels remain constant during a time slot, and independently fade slot by slot. At the beginning of each time slot, the BS randomly generates $M N_{t}$-dimensional normalized orthogonal vectors $\mathbf{w}_{m}, m=1, \cdots, M$, where $M$ with the constraint of $1 \leq M \leq N_{t}$ is the so-called transmission mode. For example, select $M$ vectors out of the $N_{t}$ singular vectors of a $N_{t} \times N_{t}$ complex Gaussian random matrix. The selection of $M$, namely mode selection, is the focus of this paper, and we will discuss it later in detail. Then, the BS broadcasts a precoded training vector $\mathbf{x}=\sum_{m=1}^{M} \mathbf{w}_{m} s_{i}$ to the SUs, where $s_{i}$ is the normalized training symbol known by the SUs in advance. It is assumed that each user has perfect CSI related to its corresponding legitimate channel through channel estimation and the information feedback is error-free. Then, the $k$-th SU computes its received signal-to-interferenceplus-noise ratio (SINR) over the $m$-th beam as

$$
\gamma_{k, m}=\frac{P\left|\mathbf{h}_{k} \mathbf{w}_{m}\right|^{2}}{\sum_{i=1, i \neq m}^{M} P\left|\mathbf{h}_{k} \mathbf{w}_{i}\right|^{2}+\sigma^{2}},
$$

where $P$ is the transmit power on each beam and $\sigma^{2}$ is the noise variance. By comparing its $M$ SINRs over $M$ beams, the $k$-th SU finds the optimal beam according to the following criterion:

$$
\mathbf{w}_{m_{k}}=\arg \max _{1 \leq m \leq M} \gamma_{k, m} .
$$

Then, the $k$-th SU conveys the index $m_{k}$ and the corresponding SINR $\gamma_{k, m_{k}}$ to the BS. After receiving the feedback information from the $K$ SUs, the BS selects an optimal SU with the largest SINR for each beam. Thereafter, the BS communicates with the selected $M$ SUs in the rest of this time slot. It is worth pointing out the probability of a SU being selected by multiple beams if the number of SU is large, so we neglect it in this paper. For convenience, we use $\mathbf{h}_{m}$ to denote the SU's channel vector over the $m$-th beam $\mathbf{w}_{m}$, then the corresponding legitimate channel capacity and the eavesdropper channel capacity are given by

$$
C_{l, m}=\log _{2}\left(1+\lambda_{m}\right),
$$

and

$$
C_{e, m}=\log _{2}\left(1+\eta_{m}\right),
$$

respectively, where $\lambda_{m}=\frac{\left|\mathbf{h}_{m} \mathbf{w}_{m}\right|^{2}}{\sum_{i=1, i \neq m}^{M}\left|\mathbf{h}_{m} \mathbf{w}_{i}\right|^{2}+\sigma^{2} / P}$ and $\eta_{m}=$ $\frac{\left|\mathbf{g w}_{m}\right|^{2}}{\sum_{i=1, i \neq m}^{M}\left|\mathbf{g w}_{i}\right|^{2}+\sigma^{2} / \alpha^{2} P}$. Thus, the secrecy rate on the $m$-th beam can be expressed as [29]

$$
C_{s e c, m}=\left(C_{l, m}-C_{e, m}\right)^{+} .
$$

Since the BS have no knowledge of the eavesdropper channel $\mathbf{g}$, it is impossible to maintain a steady secrecy rate over all realizations of the fading channel. In this case, the BS can only transmit the signal with a fixed rate. Thus, there inevitably exists the case that the transmission rate is greater than the secrecy rate. Then, the information may be intercepted by the eavesdropper. In order to guarantee the security of information transmission, the probability that the transmission rate surpasses the secrecy rate, namely the outage probability, must be controlled within a bearable range. In previous literatures, the achievable maximum secrecy rate fulfilling a given requirement on the outage probability $\varepsilon$ is called as secrecy outage capacity $R_{m}(\varepsilon)$. Mathematically, it is given by

$$
P_{r}\left(R_{m}(\varepsilon)>C_{s e c, m}\right)=\varepsilon .
$$

In this paper, we take the sum secrecy outage capacity as the performance metric. As the name implies, sum secrecy outage capacity denotes the sum of all scheduled SUs' secrecy outage capacity. Note that given a requirement on the outage probability $\varepsilon$, an important factor affecting the sum secrecy outage capacity is the number of accessing users $M$, namely transmission mode. A large $M$ means higher inter-user interference, resulting in a decrease of both the legitimate and the eavesdropper channel capacities. Therefore, there exists an optimal $M$ maximizing the sum secrecy outage capacity. In what follows, we focus on the optimal mode selection for such a MU-MIMO downlink network, so as to maximize the sum secrecy outage capacity.

\section{Optimal Mode Selection}

In this section, we concentrate on dynamic mode selection in a MU-MIMO downlink network according to channel conditions and security requirements, so as to maximize the sum secrecy outage capacity. Prior to discussing the mode selection, we first give a quantitative analysis of the secrecy outage capacity to reveal the effect of transmission mode. From (6), the outage probability on the $m$-th beam can be calculated as

$$
\begin{aligned}
\varepsilon & =P_{r}\left(R_{m}(\varepsilon)>\log _{2}\left(\frac{1+\lambda_{m}}{1+\eta_{m}}\right)\right) \\
& =P_{r}\left(\lambda_{m}<\left(1+\eta_{m}\right) 2^{R_{m}(\varepsilon)}-1\right) \\
& =\int_{0}^{\infty} \int_{0}^{\left(1+\eta_{m}\right) 2^{R_{m}(\varepsilon)}-1} f_{\lambda_{m}}(x) f_{\eta_{m}}(y) d x d y \\
& =\int_{0}^{\infty} F_{\lambda_{m}}\left((1+y) 2^{R_{m}(\varepsilon)}-1\right) f_{\eta_{m}}(y) d y
\end{aligned}
$$

where $f_{\lambda_{m}}(x)$ and $F_{\lambda_{m}}(x)$ are the probability density function (pdf) and cumulative distribution function (cdf) of $\lambda_{m}$, respectively, and $f_{\eta_{m}}(y)$ is the pdf of $\eta_{m}$. Clearly, in order to derive the outage probability, the key is to obtain the distributions of $\lambda_{m}$ and $\eta_{m}$. In the following, we turn our attention to the analysis of the two distributions.

According to the definition of $\lambda_{m}$, it can be considered as the maximum SINR on the $m$-th beam through choosing the optimal channel $\mathbf{h}_{m}$ out of $K$ ones. For an arbitrary channel vector $\mathbf{h}_{n}$ with i.i.d. zero mean and unit variance complex 
Gaussian distributed entries, $\left|\mathbf{h}_{n} \mathbf{w}_{m}\right|^{2}$ is $\chi^{2}(2)$ distributed [32], and thus $\sum_{i=1, i \neq m}^{M}\left|\mathbf{h}_{n} \mathbf{w}_{i}\right|^{2}$ is $\chi^{2}(2 M-2)$ distributed. Therefore, for a random variable $\xi=\frac{\left|\mathbf{h}_{n} \mathbf{w}_{m}\right|^{2}}{\sum_{i=1, i \neq m}^{M}\left|\mathbf{h}_{n} \mathbf{w}_{i}\right|^{2}+\sigma^{2} / P}$, its cdf $F_{\xi}(x)$ can be derived as

$$
\begin{aligned}
F_{\xi}(x) & =\int_{0}^{\infty} \int_{0}^{x\left(y+\sigma^{2} / P\right)} \frac{\exp (-y) y^{M-2}}{\Gamma(M-1)} \exp (-z) d z d y \\
& =1-\frac{\exp (-x / \rho)}{(1+x)^{M-1}}
\end{aligned}
$$

where $\rho=P / \sigma^{2}$ is the transmit SNR. Since $\lambda_{m}$ is the maximum value from $K$ independent random variables distributed as $\xi$, we have

$$
\begin{aligned}
F_{\lambda_{m}}(x) & =\left(F_{\xi}(x)\right)^{K} \\
& =\left(1-\frac{\exp (-x / \rho)}{(1+x)^{M-1}}\right)^{K}
\end{aligned}
$$

For $\eta_{m}$, since the selection of $\mathbf{w}_{m}$ is independent of the eavesdropper channel $\mathbf{g}$, so it has the cdf similar to $\xi$, and thus its pdf can be expressed as

$$
\begin{aligned}
f_{\eta_{m}}(y) & =\frac{\partial\left(1-\frac{\exp \left(-y / \alpha^{2} \rho\right)}{(1+y)^{M-1}}\right)}{\partial y} \\
& =\frac{(M-1) \exp \left(-y / \alpha^{2} \rho\right)}{(1+y)^{M}}+\frac{\exp \left(-y / \alpha^{2} \rho\right)}{\alpha^{2} \rho(1+y)^{M-1}}(1.0)
\end{aligned}
$$

Substituting (9) and (10) into (7), the outage probability is transformed as (11) at the top of next page, where $a(n)=$ $\frac{\exp \left(-n\left(2^{R_{m}(\varepsilon)}-1\right) / \rho\right)}{2^{n(M-1) R_{m}(\varepsilon)}}, \mu(n)=\left(n 2^{R_{m}(\varepsilon)}+1 / \alpha^{2}\right) / \rho, \nu(n)=$ $n(M-1)+M$ and $v(n)=(n+1)(M-1)$. 111 is derived according to [27, Eqn. 3.3532]. $W(x, N)$ with $N$ being a natural number, is defined as

$$
W(x, N)= \begin{cases}1 / x & \text { if } N=0 \\ -\exp (x) \mathrm{E}_{\mathrm{i}}(-x) & \text { if } N=1 \\ \frac{1}{\Gamma(N)} \sum_{n=1}^{N-1} \Gamma(n)(-x)^{N-1-n} & \\ -\frac{(-x)^{N-1}}{\Gamma(N)} \exp (x) \mathrm{E}_{\mathrm{i}}(-x) & \text { if } N \geq 2\end{cases}
$$

where $\mathrm{E}_{\mathrm{i}}(x)=\int_{-\infty}^{x} \frac{\exp (t)}{t} d t$ is the exponential integral function. Since $\varepsilon$ is a monotonously increasing function of $R_{m}(\varepsilon)$, for a given $\varepsilon$, it is able to find the associated $R_{m}(\varepsilon)$ with a certain transmission mode $M$ according to (11). For convenience, we use $G\left(R_{m}(\varepsilon)\right)$ to represent (11), and thus $G^{-1}(\varepsilon)$ is equivalent to $R_{m}(\varepsilon)$, where $G^{-1}(x)$ indicates the inversive function of $G(x)$. From (11), we can also derive the interception probability that the secrecy rate is less than zero by letting $R_{m}(\varepsilon)=0$. Mathematically, it can be expressed as (12) at the top of next page.

For such a homogeneous network, if the SUs have a common requirement on the outage probability $\varepsilon$, the sum secrecy outage capacity with transmission mode $M$ is given by

$$
R=M G^{-1}(\varepsilon) .
$$

In this paper, we intend to select an optimal transmission mode $M^{\star}$, so as to maximize the sum secrecy outage capacity. However, due to the complexity of (11), it is difficult to present an explicit expression for $M^{\star}$. As we know, given the number of BS antennas $N_{t}$, the transmission mode $M$ belongs to $\left[1, N_{t}\right]$. In practical systems, the number of BS antennas is quite limited, i.e., $N_{t}=4$ in LTE systems and $N_{t}=8$ in LTE-A systems. Thus, we could first derive the secrecy outage capacity for each mode, then determine the optimal mode with the maximum sum secrecy outage capacity. The whole procedure can be summarized as below.

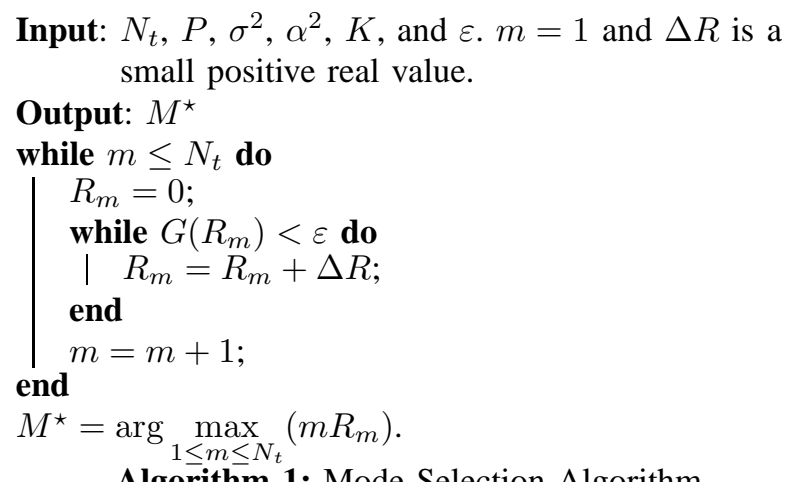

Remark: Through mode selection, we find the optimal tradeoff among spatial multiplexing gain, inter-user interference and anti-eavesdropping, so the sum secrecy outage capacity is maximized. As a simple example, if the SNR is sufficiently high, multiuser transmission may lead to performance saturation due to inter-user interference, so the secrecy performance is impossible to be improved by increasing the SNR. In this context, single user transmission, namely $M=1$, may be optimal in the sense of maximizing the sum secrecy outage capacity. Moreover, it is worth pointing out that Algorithm 1 can be extended to a general case with an arbitrary detection technique. Specifically, for a certain detection technique, such as successive interference cancellation, we can derive the corresponding sum secrecy outage capacity or the other secrecy performance metrics, which is always a function of the transmission mode. Similarly, by optimizing the secrecy performance, it is possible to obtain the optimal transmission mode.

\section{Asymptotic AnALYSis}

In order to reduce the complexity of mode selection, we perform asymptotical analysis in some extreme cases, such as noise-limited case, interference-limited case and large SU number case. In what follows, we investigate the three cases, respectively.

\section{A. Noise-Limited Case}

If transmit power $P$ is quite small or the noise variance $\sigma^{2}$ is large enough, the interference terms of $\lambda_{m}$ and $\eta_{m}$ can be 


$$
\begin{aligned}
\varepsilon= & \int_{0}^{\infty}\left(1-\frac{\exp \left(-\left(2^{R_{m}(\varepsilon)}-1\right) / \rho\right) \exp \left(-\left(2^{R_{m}(\varepsilon)} / \rho\right) y\right)}{2^{(M-1) R_{m}(\varepsilon)}(1+y)^{M-1}}\right)^{K} \frac{(M-1) \exp \left(-y / \alpha^{2} \rho\right)}{(1+y)^{M}} d y \\
& +\int_{0}^{\infty}\left(1-\frac{\exp \left(-\left(2^{R_{m}(\varepsilon)}-1\right) / \rho\right) \exp \left(-\left(2^{R_{m}(\varepsilon)} / \rho\right) y\right)}{2^{(M-1) R_{m}(\varepsilon)}(1+y)^{M-1}}\right)^{K} \frac{\exp \left(-y / \alpha^{2} \rho\right)}{\alpha^{2} \rho(1+y)^{M-1}} d y \\
= & 1+\int_{0}^{\infty} \sum_{n=1}^{K}\left(\begin{array}{c}
K \\
n
\end{array}\right)(-1)^{n}(M-1) a(n) \frac{\exp \left(-\left(\left(n 2^{R_{m}(\varepsilon)}+1 / \alpha^{2}\right) / \rho\right) y\right)}{(1+y)^{n(M-1)+M}} d y \\
& +\int_{0}^{\infty} \sum_{n=1}^{K}\left(\begin{array}{c}
K \\
n
\end{array}\right)(-1)^{n} \frac{a(n)}{\alpha^{2} \rho} \frac{\exp \left(-\left(\left(n 2^{R_{m}(\varepsilon)}+1 / \alpha^{2}\right) / \rho\right) y\right)}{(1+y)^{(n+1)(M-1)}} d y \\
= & 1+\sum_{n=1}^{K}\left(\begin{array}{c}
K \\
n
\end{array}\right)(-1)^{n}\left((M-1) a(n) W(\mu(n), \nu(n))+\frac{a(n)}{\alpha^{2} \rho} W(\mu(n), v(n))\right) . \\
P_{r}\left(C_{s e c, m}<0\right)= & G(0) \\
= & 1-\sum_{n=1}^{K}\left(\begin{array}{c}
K \\
n
\end{array}\right)(-1)^{n}\left((M-1) W\left(\left(n+1 / \alpha^{2}\right) / \rho, \nu(n)\right) \frac{1}{\alpha^{2} \rho} W\left(\left(n+1 / \alpha^{2}\right) / \rho, v(n)\right)\right) .
\end{aligned}
$$

negligible with respect to the noise term, namely the noiselimited case. In this case, we obtain a simple mode selection scheme as follows:

Theorem 1: For the noise-limited case, full spatial multiplexing, namely $M^{\star}=N_{t}$, can obtain the maximum sum secrecy outage capacity.

Proof: Please refer to Appendix I.

Remark: From the Theorem 1, it is known that at extreme low SNR regime, the sum secrecy outage capacity with different numbers of SUs and/or different requirements on the outage probabilities asymptotically approaches zero as the SNR decreases. This is because as the SNR tends to zero, both the legitimate and eavesdropper channel rates approaches to zero, then the secrecy rate becomes zero regardless of the number of SUs and the requirement on the outage probability.

Moreover, the interception probability that the secrecy rate is less than zero in such a case can be expressed as (14). As $K$ approaches infinity, the interception probability in (14) approaches zero, so the probability of nonzero secrecy rate is nearly equal to 1 . Then, as long as $K$ is large enough, there is nonzero secrecy rate with probability 1 .

\section{$B$. Interference-Limited Case}

If the transmit power $P$ is quite large or the noise variance $\sigma^{2}$ is sufficiently small, the noise term is negligible with respect to the interference term in the received SINRs $\lambda_{m}$ and $\eta_{m}$, namely the interference-limited case. In this case, we also have a simple mode selection scheme as follows:

Theorem 2: For the interference-limited case, single SU transmission mode, namely $M^{\star}=1$, can obtain the maximum sum secrecy outage capacity.

Proof: Please refer to Appendix II.

Remark: From the Theorem 2, it is known that at extreme high SNR regime, the sum secrecy outage capacity with $M>$
1 asymptotically approaches zero as the SNR increases. This is because at high SNR, both the legitimate and eavesdropper channel rates with $M>1$ have the same performance ceiling due to interference limitation. In this context, the sum secrecy outage capacity tends to zero.

In such a case, the interception probability is given by

$$
P_{r}\left(C_{s e c, m}<0\right)=\frac{1}{K+1} .
$$

More interestingly, it is found that the interception probability $P_{r}\left(C_{s e c, m}<0\right)$ is independent of the channel condition, and is a monotonously decrease function of $K$. Then, it is possible to enhance wireless security by adding the SUs.

\section{Large SU number case}

When the number of SU $K$ is large enough, one can always find $M$ SUs with orthogonal channels, such that the inter-user interference is canceled. In this case, we present a simple mode selection scheme as follows:

Theorem 3: For the large SU number case, $M^{\star}=N_{t}$ can obtain the maximum sum secrecy outage capacity.

Proof: Please refer to Appendix III.

This large $K$ case cancels the interference completely, which is equivalent to the noise-limited case, so they have the same optimal transmission mode. Furthermore, the interception probability in this case can be derived as

$$
\begin{aligned}
P_{r}\left(C_{\text {sec }, m}<0\right) & =2^{-\left(N_{t}-1\right) \log _{2}\left(1+\rho \ln \left(K N_{t}\right)\right)} \\
& \times \exp \left(-\frac{2^{\log _{2}\left(1+\rho \ln \left(K N_{t}\right)\right)}-1}{\alpha^{2} \rho}\right)
\end{aligned}
$$

The interception probability decreases as $K$ and $N_{t}$ increase and $\alpha^{2}$ decreases. 


$$
P_{r}\left(C_{s e c, m}<0\right)=\frac{\exp \left(\frac{1+1 / \alpha^{2}}{\rho}\right)\left(1-\left(1-\exp \left(-\left(1+1 / \alpha^{2}\right) / \rho\right)\right)^{K+1}\right)}{K+1} .
$$

\section{SIMULATION RESULTS}

To evaluate the performance of the proposed transmission mode selection scheme for a MU-MIMO downlink network employing with physical layer security, we present several simulation results in different scenarios. For convenience, we set $N_{t}=4, \alpha^{2}=0.01, K=10, \varepsilon=0.05$ and $\Delta R=0.01$ for all simulation scenarios without explicit explanation. In the following, we use AMS to denote the proposed adaptive mode selection scheme, and use FTM1 and FTM2 to denote the traditional fixed transmission mode schemes with $M=1$ and $M=N_{t}$, respectively. In addition, we use TSNR in $\mathrm{dB}$ to represent the transmit SNR $10 \log _{10} \frac{P}{\sigma^{2}}$. Without loss of generality, we take the sum secrecy outage capacity as the performance metric.

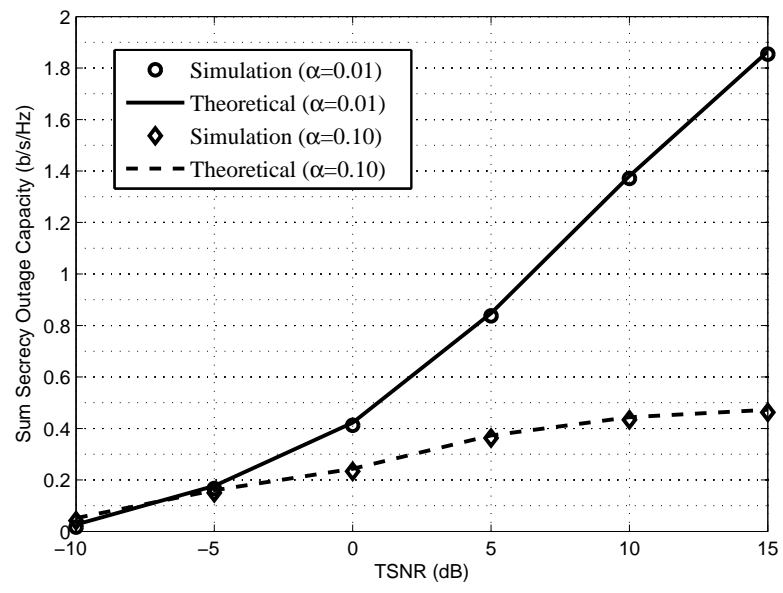

Fig. 2. Theoretical and simulation performance comparison with different path losses.

First, we check the accuracy of the derived theoretical results with different path losses. As shown in Fig 2 , the theoretical results are well coincided with the simulation results in the whole TSNR region. It is found that at low TSNR, the sum secrecy outage capacities with different path losses are nearly the same. This is because the sum secrecy outage capacity asymptotically tends to zero under this condition. However, as TSNR increases, the sum secrecy outage capacity with $\alpha=0.01$ is obviously better than that with $\alpha=0.10$, since the interception capability of the eavesdropper becomes weak. So far, short-distance interception is still an open issue for physical layer security.

Then, we compare the sum secrecy outage capacities of AMS, FTM1 and FTM2 schemes. As seen in Tab】 at the top of next page, at low SNR regime, the proposed AMS scheme chooses high order transmission mode, since the inter-user interference is quite small with respect to the noise in legitimate

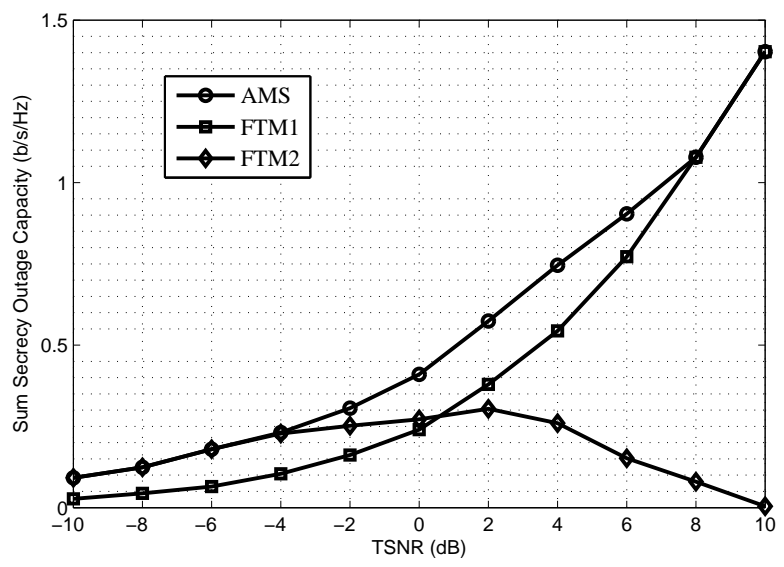

Fig. 3. Performance comparison of different mode selection schemes.

TABLE II

TRANSMISSION MODES FOR AMS WITH DIFFERENT OUTAGE PROBABILITIES.

\begin{tabular}{|c|c|c|c|c|c|c|c|c|c|c|c|}
\hline$\varepsilon_{\varepsilon} \quad$ TSNR & -10 & -8 & -6 & -4 & -2 & 0 & 2 & 4 & 6 & 8 & 10 \\
\hline 0.10 & 4 & 4 & 4 & 4 & 3 & 3 & 2 & 2 & 2 & 2 & 1 \\
\hline 0.05 & 4 & 4 & 3 & 3 & 3 & 2 & 2 & 2 & 2 & 1 & 1 \\
\hline 0.01 & 1 & 1 & 1 & 1 & 1 & 1 & 1 & 1 & 1 & 1 & 1 \\
\hline
\end{tabular}

channel, and a large $M$ can exploit the spatial multiplexing gain and counteract the interception, so as to improve the sum secrecy outage capacity. For example, as seen in Fig 3 , there is about $0.2 \mathrm{~b} / \mathrm{s} / \mathrm{Hz}$ performance gain over FTM1 with SNR $=-2 \mathrm{~dB}$. When SNR is greater than a threshold, such as $8 \mathrm{~dB}$, the proposed AMS scheme would adopt single SU transmission mode, this is because it is interference-limited under this condition, which is consistent with our theoretical claim in Theorem 2. Additionally, at high SNR, the sum secrecy outage capacity of FTM2 asymptotically approaches zero as claimed in Proposition 2. Thus, the proposed AMS scheme can achieve the optimal performance at the whole SNR regime, which is helpful for MU-MIMO secure communications with physical layer secrecy. In general, the proposed adaptive mode selection (AMS) scheme determines the optimal transmission mode by comparing $N_{t}$ sum secrecy outage capacities, while FIM1 and FIM2 uses fixed transmission modes regardless of channel conditions. Thus, AMS has relative higher complexity than FIM1 and FIM2. However, since mode selection is performed only when channel conditions change, not within each time slot, the complexity of AMS is bearable in practical systems. In addition, Theorem 1 and 2 can be used to determine the transmission mode at low and high SNR regimes respectively, which have the same complexity as FIM1 and FIM2.

Next, we investigate the impact of the outage probability 
TABLE I

TRANSMISSION MODES FOR DIFFERENT SCHEMES.

\begin{tabular}{|c|c|c|c|c|c|c|c|c|c|c|c|}
\hline Scheme TSNR & -10 & -8 & -6 & -4 & -2 & 0 & 2 & 4 & 6 & 8 & 10 \\
\hline AMS & 4 & 4 & 3 & 3 & 3 & 2 & 2 & 2 & 2 & 1 & 1 \\
\hline FTM1 & 1 & 1 & 1 & 1 & 1 & 1 & 1 & 1 & 1 & 1 & 1 \\
\hline FTM2 & 4 & 4 & 4 & 4 & 4 & 4 & 4 & 4 & 4 & 4 & 4 \\
\hline
\end{tabular}

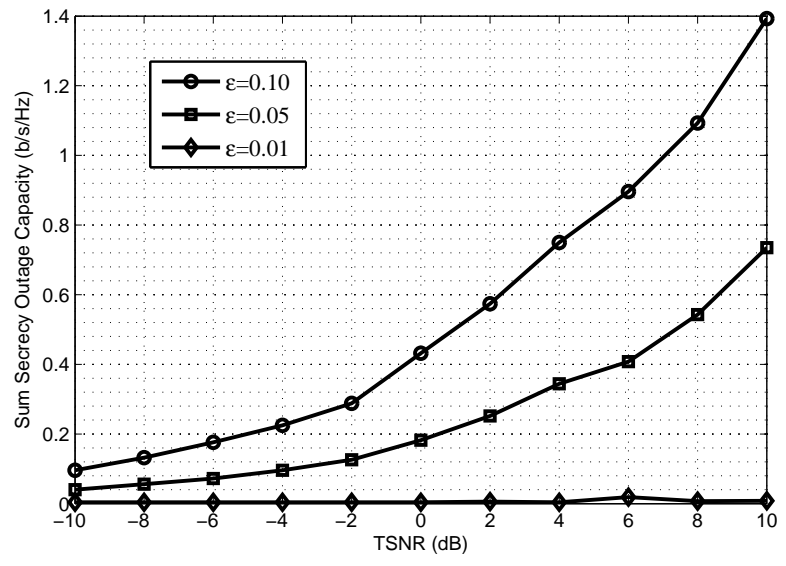

Fig. 4. Performance comparison of AMS scheme with different outage probabilities.

TABLE III

TRANSMISSION MODES FOR AMS WITH DIFFERENT NUMBERS OF SUS.

\begin{tabular}{|c|c|c|c|c|c|c|c|c|c|c|c|}
\hline$K$ & -10 & -8 & -6 & -4 & -2 & 0 & 2 & 4 & 6 & 8 & 10 \\
\hline 50 & 4 & 4 & 4 & 4 & 3 & 2 & 2 & 2 & 2 & 2 & 2 \\
\hline 20 & 4 & 4 & 4 & 4 & 3 & 2 & 2 & 2 & 2 & 2 & 1 \\
\hline 5 & 4 & 4 & 4 & 3 & 2 & 2 & 2 & 1 & 1 & 1 & 1 \\
\hline
\end{tabular}

on the sum secrecy outage capacity of the proposed AMS scheme. The outage probability $\varepsilon$ represents the interception probability with a given secrecy rate. As shown in Fig, 4, when $\varepsilon=0.01$, namely a quite small interception probability, the feasible secrecy outage capacity is nearly equal to zero. As $\varepsilon$ increases, the sum secrecy outage capacity improves accordingly. For example, one with $\varepsilon=0.10$ has about 0.5 $\mathrm{b} / \mathrm{s} / \mathrm{Hz}$ performance gain over the one with $\varepsilon=0.05$ at SNR $=6 \mathrm{~dB}$. Furthermore, the performance gain becomes larger with the increase of SNR. Note that the sum secrecy outage capacities with different outage probabilities approaches zero at low SNR regime, which reconfirms the claims in Proposition 1. From Tab it is seen that as the requirement on the outage probability relaxes, AMS will be apt to choose a high order transmission mode, especially at low SNR regime.

Finally, we show the benefit of the proposed AMS scheme from the perspective of the SU number. As seen in Fig 5, with the increase of the SU number $K$, the sum secrecy outage capacity increases accordingly, since the probability that the channels of the selected SUs are orthogonal becomes larger. In other words, the inter-user interference in legitimate channel is smaller gradually while the one in the eavesdropper channel

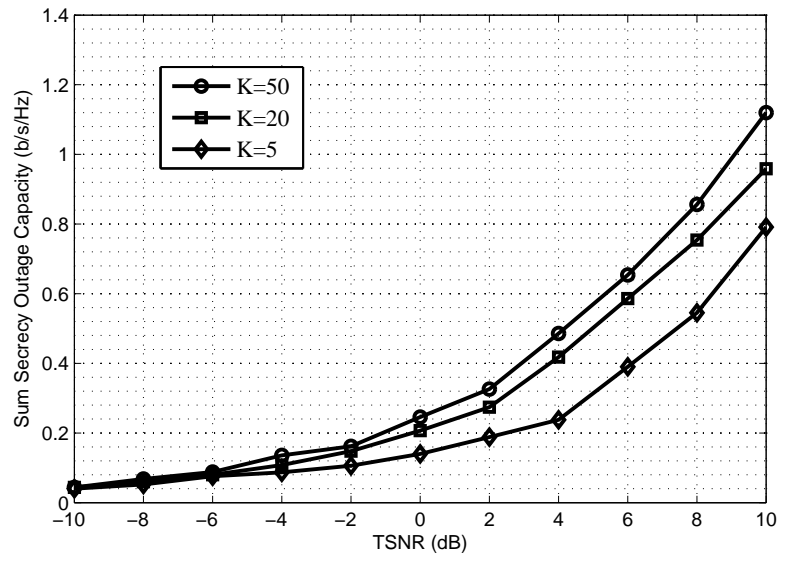

Fig. 5. Performance comparison of AMS scheme with different number of SUs.

remains unchaged in the statistical sense. In addition, it is found that the performance gain by increasing $K$ becomes smaller with a large $K$. For example, at SNR $=8 \mathrm{~dB}$, the performance gain by increasing 15 SUs from $K=5$ to $K=$ 20 heavily surpasses that by increasing 30 SUs from $K=20$ to $K=50$. It is because when $K$ is large, the received SNR based on OSDMA approaches $\rho \ln \left(K N_{t} N_{r}\right)$ as analyzed in Section IV.C, so the gain becomes smaller by increasing the same SUs as $K$ increases. Moreover, the performance gain by adding SUs is negligible at low SNR, which proves Proposition 1 again. Similarly, from Tab!II, it is also seen that as the number of SUs increases, AMS will be apt to choose a high order transmission mode, especially at low SNR regime.

\section{CONCLUSION}

The main contribution of this paper is to exploit the benefit of inter-user interference to improve the sum secrecy outage capacity in MU-MIMO downlink networks employing physical layer security. It is found that under different channel conditions, the inter-user interference plays different roles. Therefore, we propose an effectively adaptive transmission mode selection scheme maximizing the sum secrecy outage capacity. Furthermore, asymptotic analysis is carried out to further insights on mode selection. For example, our asymptotical results show that at low SNR regime, maximum available mode should be adopted. Both relaxing the requirement on the outage probability and increasing the number of SUs are hardly helpful to improve the secrecy performance. On the contrary, at high SNR regime, single SU mode is the best choice and multiple-SU mode will result in zero rate. 


\section{APPENDIX A}

\section{PROOF OF THEOREM 1}

In the noise-limited case, the cdf of $\lambda_{m}$ and the pdf of $\eta_{m}$ are reduced to

$$
F_{\lambda_{m}}(x)=\left(1-\exp \left(-\frac{x}{\rho}\right)\right)^{K}
$$

and

$$
f_{\eta_{m}}(y)=\frac{1}{\alpha^{2} \rho} \exp \left(-\frac{y}{\alpha^{2} \rho}\right),
$$

respectively. Substituting (17) and (18) into (7), the outage probability in this case can be computed as

$$
\begin{aligned}
\varepsilon= & \int_{0}^{\infty}\left(1-\exp \left(-\frac{2^{R_{m}(\varepsilon)}-1}{\rho}\right) \exp \left(-\frac{2^{R_{m}(\varepsilon)}}{\rho} y\right)\right)^{K} \\
& \times \frac{\exp \left(-\frac{y}{\alpha^{2} \rho}\right)}{\alpha^{2} \rho} d y \\
= & 1+\sum_{n=1}^{K}\left(\begin{array}{c}
K \\
n
\end{array}\right)(-1)^{n} \frac{\exp \left(-n\left(2^{R_{m}(\varepsilon)}+1 / \alpha^{2}\right) / \rho\right)}{n 2^{R_{m}(\varepsilon)}+1}(19)
\end{aligned}
$$

Interestingly, it is found that the outage probability in (19) is independent of the transmission mode. Under this condition, $M=N_{t}$ can achieve the full multiplexing gain, and also leads to the maximum sum secrecy outage capacity. Thus, we complete the proof.

\section{APPENDIX B \\ PROOF OF THEOREM 2}

In the interference-limited case, the cdf of $\lambda_{m}$ and the pdf of $\eta_{m}$ can be expressed as

$$
F_{\lambda_{m}}(x)=\left(1-\frac{1}{(1+x)^{M-1}}\right)^{K},
$$

and

$$
f_{\eta_{m}}(y)=\frac{M-1}{(1+y)^{M}} .
$$

Similarly, the outage capacity is given by

$$
\begin{aligned}
\varepsilon & =\int_{0}^{\infty}\left(1-\frac{1}{2^{(M-1) R_{m}(\varepsilon)}(1+y)^{M-1}}\right)^{K} \frac{M-1}{(1+y)^{M}} d y \\
& =1+\sum_{n=1}^{K}\left(\begin{array}{c}
K \\
n
\end{array}\right) \frac{\left(-2^{-(M-1) R_{m}(\varepsilon)}\right)^{n}}{n+1} \\
& =\frac{2^{(M-1) R_{m}(\varepsilon)}\left(1-\left(1-2^{-(M-1) R_{m}(\varepsilon)}\right)^{K+1}\right)}{K+1} \\
& =\frac{2^{\frac{M-1}{M} R}\left(1-\left(1-2^{-\frac{M-1}{M} R}\right)^{K+1}\right)}{K+1} .
\end{aligned}
$$

where (22) is obtained according to [27, Eqn.0.1553]. From (23), it is known that $\varepsilon$ is a monotonously increasing function of $\frac{M-1}{M}$ and $R$. Given a requirement on the outage probability $\varepsilon$, the sum secrecy outage capacity $R$ is maximized by minimizing $\frac{M-1}{M}$. Clearly, $\frac{M-1}{M}$ is minimized when $M=1$, which proves the claims in Theorem 2.

\section{APPENDIX C Proof of THEOREM 3}

If the user number $K$ is large enough, there is the following important property that $\lambda_{m} \rightarrow \rho \ln \left(K N_{t}\right)$ [34]. In other words, the legitimate channel capacity approaches a constant $\log _{2}(1+$ $\left.\rho \ln \left(K N_{t}\right)\right)$, so the outage probability is transformed as

$$
\begin{aligned}
\varepsilon & =P_{r}\left(\eta_{m}>2^{\log _{2}\left(1+\rho \ln \left(K N_{t}\right)\right)-R_{m}(\varepsilon)}-1\right) \\
& =\frac{\exp \left(-\frac{2^{\log _{2}\left(1+\rho \ln \left(K N_{t}\right)\right)-R_{m}(\varepsilon)}-1}{\alpha^{2} \rho}\right)}{\left(2^{\log _{2}\left(1+\rho \ln \left(K N_{t}\right)\right)-R_{m}(\varepsilon)}\right)^{M-1}} .
\end{aligned}
$$

As seen in (24), $\varepsilon$ is a monotonously increasing function of $R_{m}(\varepsilon)$ and is a monotonously decreasing function of $M$. Given a requirement on the outage capacity $\varepsilon$, secrecy outage capacity $R_{m}(\varepsilon)$ is maximized with $M=N_{t}$, which also achieves the full multiplexing gain. Thus, we get Theorem 3.

\section{REFERENCES}

[1] A. D. Wyner, "The wire-tap channel," Bell Syst. Tech. J., vol. 54, pp. 1355-1387, Oct. 1975.

[2] P. K. Gopala, L. Lai, and H. El. Gamal, "On the secrecy capacity of fading channels," IEEE Trans. Inf. Theory, vol. 54, no. 10, pp. 4687-4698, Oct. 2008.

[3] F. Oggier, and B. Hassibi, "The secrecy capacity of the MIMO wiretap," IEEE Trans. Inf. Theory, vol. 57, no. 8, pp. 4961-4972, Feb. 2011.

[4] E. Ekrem, and S. Ulukus, "The secrecy capacity region of the Gaussian MIMO multi-receiver wiretap channel," IEEE Trans. Inf. Theory, vol. 57, no. 4, pp. 2083-2114, Feb. 2011.

[5] T. Liu, and S. Shamai, "A note on the secrecy capacity of the multipleantenna wiretap channel," IEEE Trans. Inf. Theory, vol. 55, no. 6, pp. 2547-2553, Jun. 2009.

[6] A. Khisti, and G. W. Wornell, "Secure transmission with multiple antennapart II: the MIMOME wiretap channel," IEEE Trans. Inf. Theory, vol. 56, no. 11, pp. 5515-5532, Nov. 2010.

[7] X. Chen, and L. Lei, "Energy-efficient optimization for physical layer security in multi-antenna downlink networks with QoS guarantee," IEEE Commun. Lett., vol. 17, no. 4, pp. 637-640, Apr. 2013.

[8] A. Mukherjee, and A. L. Swindlehurst, "Robust beamforming for security in MIMO wiretap channels with imperfect CSI," IEEE Trans. Signal Process., vol. 59, no. 1, pp. 351-361, Jan. 2011.

[9] C. Zhang, H. Gao, T. Lv, Y. Lu, and X. Su, "Beamforming to secure two-way relay networks with physical layer network coding," in Proc. IEEE GLOBECOM, pp. 1-6, Dec. 2014.

[10] J. Chen, X. Chen, T. Liu, and L. Lei, "Energy-efficient power allocation for secure communications in large-scale MIMO relaying systems," in Proc. IEEE ICCC, pp. 1-6, Oct. 2014.

[11] C. Zhang, H. Gao, H. Liu, and T. Lv, "Robust beamforming and jamming for secure AF relay networks with multiple eavesdroppers," in Proc. IEEE Milcom, pp. 495-500, Oct. 2014.

[12] A. Khisti, and G. W. Wornell, "Secure transmission with multiple antennas I: the MISOME wiretap channel," IEEE Trans. Inf. Theory, vol. 56, no. 7, pp. 3088-3104, Jul. 2010.

[13] T. V. Nguye, and H. Shin, "Power allcoation and achievable secrecy rates in MISOME wiretap channels," IEEE Commun. Lett., vol. 15, no. 11, pp. 1196-1198, Nov. 2011.

[14] M. Bloch, J. Barros, M. Rodrigues, and S. Mclaughlin, "Wireless information-theoretic security," IEEE Trans. Inf. Theory, vol. 54, no. 6, pp. 2515-2534, Jun. 2008.

[15] H. Alves, R. D. Souza, M. Debbah, and M. Bennis, "Performance of transmit antenna selection physical layer security schemes," IEEE Signal Process. Lett., vol. 19, no. 6, pp. 372-375, Jun. 2012.

[16] N. Yang, H. A. Suraweera, I. B. Collings, and C. Yuen, "Physical layer security of TAS/MRC with antenna correlation," IEEE Trans. Inf. Forensics and Security, vol. 8, no. 1, pp. 254-259, Jan. 2013. 
[17] X. Chen, and R. Yin, "Performance analysis for physical layer security in multi-antenna downlink networks with limited CSI feedback," IEEE Wireless Commun. Lett., vol. 2, no. 5, pp. 503-506, Oct. 2013.

[18] B. Shafi, Z. Ding, and G. Y. Li, "On secrecy of codebook-based transmission beamforming under receiver limited feedback," IEEE Trans. Wireless Commun., vol. 10, no. 4, pp. 1212-1223, Apr. 2011.

[19] K. Huang, R. W. Heath, Jr., and J. G. Andrews, "Space division multiple access with a sum feedback rate constraint," IEEE Trans. Signal Process., vol. 55, no. 7, pp. 3879-3891, Jul. 2007.

[20] C. Yuen, and B. M. Hochwald, "Achieving near-capacity at low SNR on a multiple-antenna multiple-user channel," IEEE Trans. Commun., vol. 57, no. 1, pp: 69-74, Jan 2009.

[21] X. Chen, and H-H. Chen, "Physical layer security in multi-cell MISO downlink with incomplete CSI-a unified secrecy performance analysis," IEEE Trans. Signal Process., vol. 62, no. 23, pp. 6286-6297, Dec. 2014

[22] J. Zhang, C. Yuen, C.-K. Wen, S. Jin, and X. Gao, "Ergodic secrecy sum-rate for multiuser downlink transmission via regularized channel inversion: large system analysis," IEEE Comms Lett., vol. 8, no. 9, pp. 1627-1630, July 2014.

[23] A. Khisti, A. Tchamkerten, and G. W. Wornell, "Secure broadcasting over fading channels," IEEE Trans. Inf. Theory, vol. 54, no. 6, pp. 24532469, Sep. 2008.

[24] M. Pei, J. Wei, K-K. Wong, and X. Wang, "Masked beamforming for multiuser MIMO wiretap channels with imperfect CSI," IEEE Trans. Wireless Commun., vol. 11, no. 2, pp. 544-549, Feb. 2012.

[25] X. Chen, Z. Zhang, S. Chen, and C. Wang, "Adaptive mode selection for multiuser MIMO downlink employing rateless codes with QoS provisioning," IEEE Trans. Wireless Commun., vol. 11, no. 2, pp. 790799, Feb. 2012

[26] J. Zhang, M. Kountouris, J. G. Andrews, and R. W. Heath Jr., "Multimode transmission for the MIMO broadcast channel with imperfect channel state information," IEEE Trans. Commun., vol. 59, no. 3, pp. 803-814, Mar. 2011.

[27] A. Mukherjee, S. A. A. Fakoorian, J. Huang, and A. L. Swindlehurst, "Principles of physical layer security in multiuser wireless networks: a survey," IEEE Commun. Surveys \& Tutorials, vol. 16, no. 3, pp. 15501573, Sept. 2014

[28] M. Pei, A. L. Swindlehurst, D. Ma, and J. Wei, "On ergodic secrecy rate for MISO wiretap broadcast channels with opportunistic scheduling," IEEE Commun. Lett., vol. 18, no. 1, pp. 50-53, Jan. 2014.

[29] I. Krikidis, and B. Ottersten, "Secrecy sum-rate for orthogonal random beamforming with opportunistic scheduling," IEEE Signal Process. Lett., vol. 20, no. 2, pp. 141-144, Feb. 2013.

[30] C. Wan, A. Forenza, J. G. Andrews, R. W. Heath, Jr., "Opportunistic space-division multiple access with beam selection," IEEE Trans. Commun., vol. 55, no. 12, pp. 2371-2380, Sep. 2007.

[31] S. Sanayei, and A. Nosratinia, "Opportunistic downlink transmission with limited feedback," IEEE Trans. Inf. Theory, vol. 53, no. 11, pp. 4363-4362, Nov. 2007.

[32] K. K. Mukkavilli, A. Sabharwal, E. Erkip, and B. Aazhang, "On beamforming with finite rate feedback in multiple-antenna systems," IEEE Trans. Inf. Theory, vol. 49, no. 10, pp. 2562-2579, Oct. 2003.

[33] I. S. Gradshteyn, and I. M. Ryzhik, "Tables of intergrals, series, and products," Acedemic Press, USA, 2007.

[34] P. Viswanath, D. N. C. Tse, and R. Laroia, "Opportunistic beamforming using dumb antennas," IEEE Trans. Inf. Theory, vol. 48, no. 6, pp. 12771294, Jun. 2002. 